\title{
Why Do Black-Hole X-ray Binaries Tend to Be Transient?
}

\author{
Jean-Pierre Lasota \\ Institut d'Astrophysique de Paris \\ 98bis Bd Arago, 75014 Paris, France
}

\begin{abstract}
Black hole X-ray binaries are transient probably because their discs are subject to the same thermal-viscous instability which is present in dwarf nova binary systems. I discuss applications of the dwarf-nova instability model to transient, low-mass, X-ray binary systems. When disc truncation and X-ray irradiation are taken into account this model is capable of reproducing the basic properties of $\mathrm{X}$-ray binary outbursts ([3]).
\end{abstract}

\section{The thermal-viscous instability}

All Low Mass X-ray Binaries (LMXBs) in which the presence of a black hole is inferred from dynamical mass determination are transient. This could mean that all Black Hole LMXBs (BHLMXBs) are transient, but there could be non-transient systems in which black holes have masses in the range allowed for neutron stars. For this reason it is more prudent to say that BHLMXBs 'tend' to be transient (as suggested by the organizers of this meeting) rather than to affirm that all of them are transient.

The responsibility for outbursts in low mass, close binary systems is commonly imputed (e.g. 222]) to the usual suspect: the thermal-viscous instability present in discs of a subclass of cataclysmic variable stars: the dwarf-nova systems. There, for temperatures corresponding to hydrogen recombination, changes in opacity (emissivity) strongly affect cooling mechanism dependence on temperature and lead to a thermal instability under the standard assumption about viscous heating. This instability is intimately related to a viscous instability. A disc's thermal equilibria, at a given distance from the center, form a characteristic $S$ shape in the surface density - effective temperature plane. The middle branch of the $S$ corresponds to unstable solutions, the upper one to hot, almost fully ionized configurations, while the lower branch contains cold, quasi-neutral states. (The existence of the cold branch does not depend on the presence of convection, contrary to erroneous assertions in recent lectures on the subject [18]. In fact some attempts to modify the viscosity prescription required switching off the convection in order to get a sufficient amplitude of the jump between the cold and hot branches [2]). If the rate at which the mass is transferred from the black-hole's stellar companion is such that the disc is (somewhere) unstable, a limit cycle behaviour will follow taking the system through a cycle of oscillations between hot and cold 
states. Such a cycle is supposed to explain dwarf nova and transient LMXB outburst cycles.

The instability and the limit cycle do not, by themselves, produce outbursts similar to the ones observed in dwarf novae and transient systems. The thermal instability creates temperature gradients which propagate through the disc in the form of heating (transition to the hot branch) and cooling (transition to the cold branch) fronts. The form of the resulting luminosity variations depends on what is assumed about the viscosity or, more precisely, what is assumed about the viscosity parameter $\alpha$ appearing in the kinematic viscosity prescription $\nu=\alpha c_{s}^{2} / \Omega_{K}$, where $c_{s}$ is the speed of sound and $\Omega_{K}$ is the Keplerian frequency. Is $\alpha$ is assumed to be constant, then, because of insufficient temperature and density contrasts between the cold and hot states, the resulting light-curve has the form of regular, small amplitude outbursts with no resemblance to dwarf-nova outbursts or X-ray binary transient events. In order to obtain dwarf-nova type outbursts the viscosity parameter $\alpha$ must be assumed to be larger in the hot than in the cold state [24. This einsatz has not been, for the moment, based on any model of viscosity, although a recent argument in favour of different values of $\alpha$ in hot and cold states [8] is based on such a model (beware, however of the erroneous interpretation in $|18|$ : it applies hot equilibrium disc solutions to describe the properties of a cold non-equilibrium disc!).

The assumption of a jump in the $\alpha$ value, however, does not suffice to reproduce observed properties of outbursts in close binary systems and additional physical effects have to be added to the 'pure' disc instability model (see e.g. 26] [10]). Some of these effects, such as inner disc truncation 19] 20] and disc $\mathrm{X}$-ray irradiation [3] [4], which are important for low mass $\mathrm{X}$-ray transients, will be discussed in this article.

Figure 1 shows that the parameters of all BHLMXBs put them well below the limit for the disc thermal stability. Here I used the stability criterion for X-ray irradiated discs as proposed by Jan van Paradijs [27. Irradiation (discussed in Sect. 3) of the outer disc by accretion-produced X-rays from the inner disc gives a stability criterion which can be expressed in the form (近, [16]):

$$
\begin{aligned}
\dot{M}_{\text {transf }}>\dot{M}_{\text {crit }}^{\text {irr }} \approx 2.0 \times 10^{15} & \left(\frac{M_{1}}{M_{\odot}}\right)^{0.5}\left(\frac{M_{2}}{M_{\odot}}\right)^{-0.2} P_{\mathrm{hr}}^{1.4} \\
& \times\left(\frac{\mathcal{C}}{5 \times 10^{-4}}\right)^{-0.5} \mathrm{~g} \mathrm{~s}^{-1}
\end{aligned}
$$

where $\dot{M}_{\text {transf }}$ is the mass-transfer rate, $M_{1}$ is the black hole mass, $M_{2}$ is the mass of the companion, $P_{\mathrm{hr}}$ the orbital period in hours. $\mathcal{C}$ is a parameter describing effects of irradiation (see 肺). Systems satisfying condition (11) are stable.

However, systems with $\dot{M}_{\text {transf }}<\dot{M}_{\text {crit }}^{\text {ir }}$ are not necessarily unstable. In fact BHLMXBs are well below the stability limit (lower than one would expect 
from evolution models [19]). They are close to the stability limit for cold discs if their discs are truncated in the inner regions. There are several arguments in favour of such a truncation.

\section{The necessity of truncation}

According to the model quiescent accretion rate must everywhere satisfy the condition (12]):

$$
\dot{M}(r)<\dot{M}_{\text {cold }}=9.510^{15} \alpha^{0.01}\left(\frac{M_{1}}{\mathrm{M}_{\odot}}\right)^{-0.89}\left(\frac{R}{10^{10} \mathrm{~cm}}\right)^{2.68} \mathrm{~g} \mathrm{~s}^{-1}
$$

For a disc extending down to the last stable orbit and typical parameters, this predicts quiescent accretion several orders of magnitude lower than observed ([15]). A disc truncated at $10^{3}-10^{4} R_{S}$ (where $R_{S}$ is the Schwarzschild radius) will solve this problem, in particular if the inner 'hole' is filled by an Advection Dominated Accretion Flow (ADAF) (e.g. [23], [11], 6]). For such truncation radii, what is left of the disc could be cold and stable as shown by Fig. (11) (where the truncation radius is scaled by the circularization radius). Also, if we take into account irradiation, disc truncation is necessary in order to obtain the light-curves required by observations ([3]).

\section{Effects of irradiation}

There is overwhelming evidence that discs in LMXBs are strongly X-ray irradiated $(28])$. In outburst, a disc's optical emission is due to X-ray reprocessing. As mentioned above, irradiation modifies the stability criterion. It also affects all the properties of the outburst cycle. Its main effect is to retard the propagation of the cooling front, leading to an exponential decay in the light-curves (14]). An example of this effect is shown in Fig. (2). A systematic study of irradiation effects will be presented in Dubus et al. [3]. It appears that most of the fundamental properties of at least one class of LMXB transient systems can be reproduced by the model if both truncation and irradiation are taken into account. The transient systems concerned are those with a fast-rise exponential decay (FRED) light curves. (See next section for the discussion of recurrence times).

In systems with more complex forms of light-curves one should also take into account (the observed) irradiation of the secondary ([7, 周).

\section{Recurrence times}

Compared with most dwarf novae, black hole X-ray transients have very long recurrence times ( $>30$ years). These also seem to be longer than the 


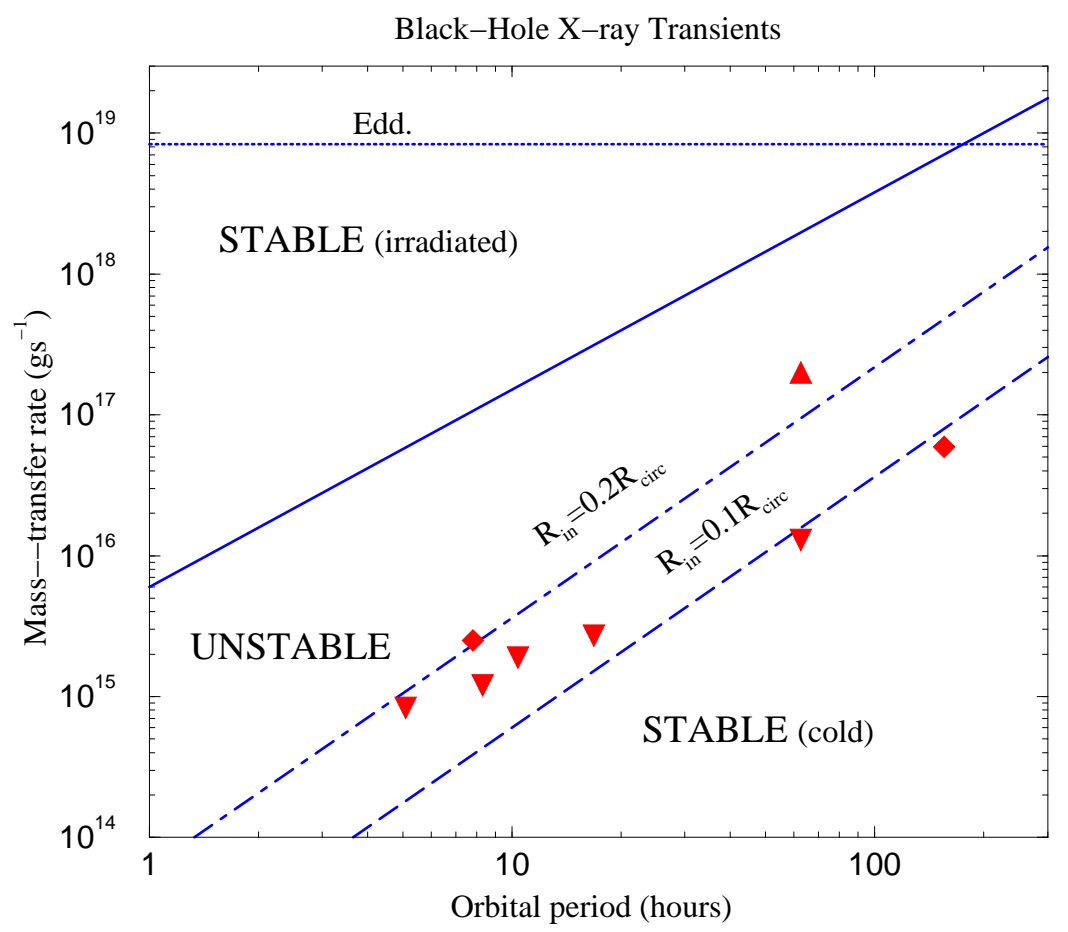

Fig. 1. Stability criteria for accretion discs in black-hole low-mass $\mathrm{X}$-ray binaries. The mass-transfer rate is estimated by dividing the mass accreted during outburst by the recurrence time. This mass accumulation rate might be slightly lower than the actual transfer rate 19. Since, except for two systems, only the lower limits on recurrence times are known, we have, for most systems, only the upper limits on the mass accumulation rate. All these systems are well below the upper line corresponding to stable irradiated discs but are rather close to the stability limit for truncated discs, represented here by two lines corresponding to truncation radii $R_{\text {in }}=0.1$ and $0.2 R_{\text {circ }}$, where $R_{\text {circ }}$ is the circularization radius. Data is taken from ref. 19]. Two values are given for GRO J1655-40: the higher one corresponds to the 1996 outburst (see []]). The dotted line marked 'Edd' corresponds to the Eddington accretion rate $\dot{M}_{\text {Edd }}=L_{\text {Edd }} / 0.1 c^{2}$ for $6 M_{\odot}$.

recurrence times of neutron star transients. Very long recurrence times can be due to several effects. First, truncated discs in these systems could be cold and stable (Fig. (11) and outbursts due to (upward) fluctuations of mass transfer. The recurrence time then would correspond to some cycle in the secondary star. Such a model was proposed for the dwarf nova WZ Sge, whose recurrence time is $\sim 30$ years $[17]$. 9 .

Second, the inner truncation radius can be such that the disc is marginally stable (see Fig. (1). Since for a stable disc the recurrence time is infinite, for a given mass-transfer rate one can always fine-tune the inner disc radius so 


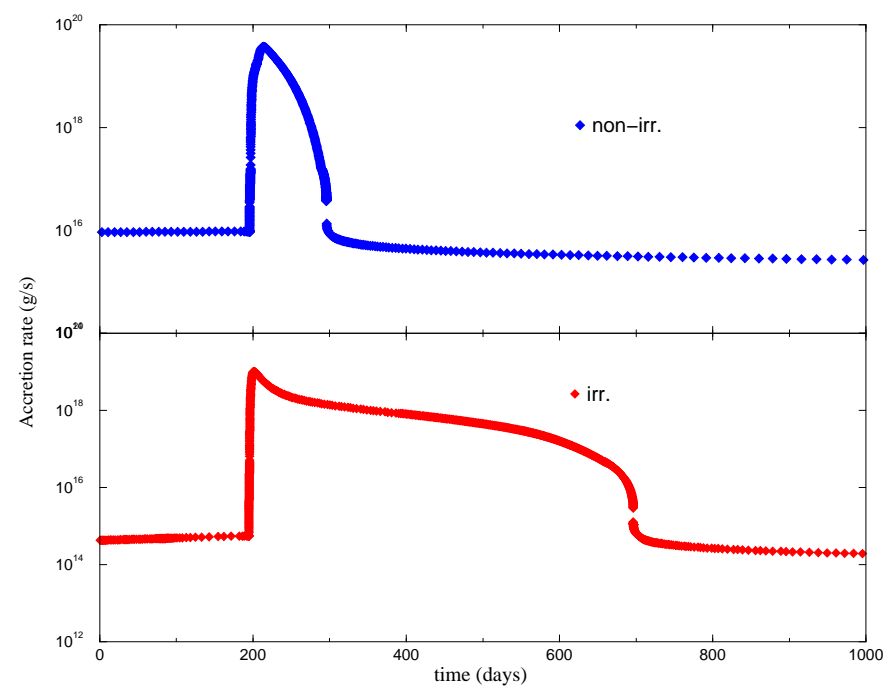

Fig. 2. Examples of lightcurves obtained in the disc instability model for truncated, non-irradiated (upper panel) and irradiated (lower panel) discs in black-hole lowmass binaries. In the non-irradiated case the cooling front rapidly propagates inwards, whereas irradiation postpones propagation of this front as suggested in 14. As a result the light curve is close to exponential, outbursts lasts longer and more mass is drained from the disc.

that the recurrence time is arbitrarily long (see e.g. 20]. This is for example the case of the model proposed in [21. Such an assumption is, however, equivalent to assuming global stability because the fine tuning would be at the mercy of even very small fluctuations of mass transfer.

Two other possibilities can be described by using an approximate formula for the recurrence time (see [25] 20])

$$
t_{\mathrm{rec}} \approx 3\left(\frac{\xi}{3}\right)\left(\frac{M_{1}}{M_{\odot}}\right)^{0.62}\left(\frac{R}{10^{10} \mathrm{~cm}}\right)^{0.14}\left(\frac{\alpha_{\mathrm{cold}}}{0.02}\right)^{-0.83}\left(\frac{T_{\mathrm{eff}}}{3000 \mathrm{~K}}\right)^{-4} \mathrm{yr}
$$

where $T_{\text {eff }}$ is the disc effective temperature, $\alpha_{\text {cold }}$ is the viscosity parameter in quiescence and $\xi$ is a factor $(3-5)$ taking into account the fact that the quiescent disc is not in viscous equilibrium[13]. In a quiescent disc $T_{\text {eff }}$ is almost independent of the radius (this is not the result of low viscosity in quiescence, contrary to the erroneous assertions in $[18]$ ).

We can see that if we assume $\alpha_{\text {cold }} \approx 10^{-3}$, Eq. (3) gives recurrence times longer than tens of years. This assumes that the quiescent effective temperature is not lower than $3000 \mathrm{~K}$. In non-irradiated discs this is indeed the case (note that irradiation is important only during the outburst) and only by lowering $\alpha_{\text {cold }}$ can one obtain long recurrence times [20]. Irradiation during outburst, however, allows one to drain more mass before the cooling 
front brings the disc into the cold state. As a result quiescent temperatures are lower than in the non- irradiated case: $T_{\text {eff }} \lesssim 2000 \mathrm{~K}$ and accordingly recurrence times are longer [3].

\section{Acknowledgments}

I am grateful to Guillaume Dubus, Anya Esin, Jean-Marie Hameury and Kristen Menou for many enlightening discussions. I thank J.-M. Hameury for data used in Fig. 2. This work was supported in part by an ASPS/CNRS grant.

\section{References}

1. Cannizzo, J.K. (1998) ApJ, 494, 318

2. Cannizzo, J.K., Chen, W., Livio, M. (1995), 454, 880

3. Dubus, G., Hameury, J.-M., Lasota, J.-P. (2000) to be submitted to A\&A

4. Dubus, G., Lasota, J.-P., Hameury, J.-M., Charles, P. (1999) MNRAS, 303, 139

5. Esin, A.A. (2000) these proceedings

6. Esin, A.A., McClintock, J.E., Narayan, R. (1997) ApJ, 489, 865

7. Esin, A.A., Lasota, J.-P., Hynes, R.I. (2000) A\&A, submitted

8. Gammie, C.F., Menou, K. (1998) ApJ, 492, L75

9. Hameury, J.-M., Lasota, J.-P., Huré, J.-M. (1997) MNRAS, 287, 937

10. Hameury, J.-M., Lasota, J.-P., Warner, B. (2000) A\&A, in press

11. Hameury, J.-M., Lasota, J.-P., McClintock, J.E., Narayan, R. (1997) ApJ, 489, 234

12. Hameury, J.-M., Menou, K., Dubus, G., Lasota, J.-P., Huré, J.-M. (1998) MNRAS, 298, 1048

13. Idan, I., Lasota, J.-P., Hameury, J.-M., Shaviv, G., (1999) Phys. Rep. 311, 213

14. King, A.R., Ritter, H. (1998) MNRAS, 293, 42

15. Lasota, J.-P. (1999) in van Paradijs, J., van den Heuvel, E.P.J, Kuulkers, E. eds., Compact Stars in Binaries, IAU Symp. 165, Kluwer, Dordrecht, p. 43

16. Lasota, J.-P. (1999) in S. Mineshige, J.C. Wheeler eds. Disk Instabilities in Close Binaries - 25 years of the Disk Instability Model, Universal Academy Press, Tokyo, p. 191

17. Lasota, J.-P., Hameury, J.-M., Huré, J.-M. (1995) A\&A, 302, 29

18. Livio, M. (1999) in J.A. Sellwood, J. Goodman eds., Astrophysical Discs - An EC Summer School, ASP Conference Series Vol. 160, p. 33

19. Menou, K., Narayan, R., Lasota, J.-P. (1999) ApJ, 513, 811

20. Menou, K., Hameury, J.-M., Lasota, J.-P., Narayan, R. (2000) MNRAS, in press

21. Meyer-Hofmeister, E., Meyer, F. (1999) A\&A, 348, 154

22. Mineshige, S., Wheeler, J.C. (1989) ApJ, 343, 241

23. Narayan, R., Barret, D., McClintock, J.E. (1997) ApJ, 482, 448

24. Smak, J. (1984) Acta Astron., 32, 101

25. Smak, J. (1993) Acta Astron., 43, 161

26. Smak, J. (1999) Acta Astron., 49, 391

27. van Paradijs, J. (1996) ApJ, 464, L139

28. van Paradijs, J., McClintock, J.E. (1995), in Lewin, W.H.G., van Paradijs, J., van den Heuvel, E.P.J. eds., X-ray Binaries, CUP, Cambridge, p. 58 